\title{
Adjuvant Chemotherapy in pT2NOMO Gastric Cancer: Findings From a Retrospective Study
}

\author{
Yu Mei ${ }^{1+}$, Xijia Feng ${ }^{2+}$, Tienan Feng ${ }^{3}$, Min Yan ${ }^{1}$, Zhenggang Zhu ${ }^{1}$, Tian $\mathrm{Li}^{4 *}$ and \\ Zhenglun Zhu ${ }^{1 *}$
}

${ }^{1}$ Department of General Surgery, Gastrointestinal Surgery, Shanghai Key Laboratory of Gastric Neoplasms, Shanghai Institute of Digestive Surgery, Ruijin Hospital, Shanghai Jiao Tong University School of Medicine, Shanghai, China, ${ }^{2}$ Department of Thoracic Surgery, Ruijin Hospital, Shanghai Jiao Tong University School of Medicine, Shanghai, China, ${ }^{3}$ Clinical Research Institute, Shanghai Jiao Tong University School of Medicine, Shanghai, China, ${ }^{4}$ School of Basic Medicine, Fourth Military Medical University, Xi'an, China

OPEN ACCESS

Edited by: Peixin Dong,

Hokkaido University, Japan

Reviewed by: Arjun Singh,

Memorial Sloan Kettering Cancer

Center, United States

Aklank Jain

Central University of Punjab, India

*Correspondence:

Tian Li

tian@fmmu.edu.cn

Zhenglun Zhu

big8424@126.com

tThese authors have contributed equally to this work

Specialty section:

This article was submitted to Pharmacology of Anti-Cancer Drugs,

a section of the journal

Frontiers in Pharmacology

Received: 29 December 2021 Accepted: 04 February 2022

Published: 17 February 2022

Citation:

Mei $Y$, Feng $X$, Feng $T$, Yan $M$, Zhu Z,

Li T and Zhu Z (2022) Adjuvant Chemotherapy in pT2NOMO Gastric

Cancer: Findings From a

Retrospective Study.

Front. Pharmacol. 13:845261.

doi: 10.3389/fphar.2022.845261
Background: There is no global consensus on adjuvant chemotherapy (ACT) for pT2NOMO gastric cancer. We conducted a retrospective study to reveal the role of $\mathrm{ACT}$ in such patients.

Methods: Patients with pT2NOMO gastric cancer who underwent radical resection with D2 lymphadenectomy for primary gastric cancer between January 2012 and May 2016 were included. Kaplan-Meier and Cox regression were used to evaluate overall survival (OS), disease-specific survival (DSS) and predictors of prognosis. Stratified analysis based on high-risk factors was conducted.

Results: Of enrolled 307 patients, 111 patients underwent surgery alone and 196 patients received ACT. Surgery alone $(H R=2.913,95 \% \mathrm{Cl}: 1.494-5.682, p=0.002)$ and total gastrectomy $(\mathrm{HR}=2.445,95 \% \mathrm{Cl}: 1.279-4.675, p=0.007)$ were independently associated with decreased OS. With the median follow-up of 73.1 months, the 5-year OS rate was $87.9 \%$ and 5 -year DSS rate was $91.8 \%$. Patients receiving ACT showed a better 5 -year OS rate $(92.9$ vs. $79.3 \%, p<0.001)$ and DSS rate $(96.8$ vs. $83.0 \%, p<0.001)$ than patients underwent surgery alone. Patients receiving monotherapy $(n=130)$ had a relatively poor prognosis compared to patients receiving dual-drug $(n=66)$ without a significant difference (92.3 vs. 93.9\%, $p=0.637$ ). In patients without high-risk factors based on the Chinese Society of Clinical Oncology (CSCO) Guidelines, ACT also provided survival benefit (96.0 vs $82.9 \%, p=0.038$ ).

Conclusions: ACT was accompanied with higher 5-year OS and DSS rates of patients with pT2NOMO gastric cancer. Patients with PT2NOMO gastric cancer, regardless of highrisk factors based on the CSCO guidelines, might be considered candidates for ACT. In regard to the therapy regimen, monotherapy might be the optimal choice, considering the adverse events.

Keywords: gastric cancer, pT2NOMO, adjuvant chemotherapy, surgery alone, prognosis 


\section{INTRODUCTION}

Gastric cancer is the fourth leading cause of death from malignant tumors worldwide and the third main cause of cancer death in China (Cao et al., 2021; Navashenaq et al., 2021; Sun et al., 2021; Zeng and Jin, 2021). Despite the incidence of gastric cancer has reduced, gastric cancer related mortality has not changed (Sukri et al., 2020; Varon et al., 2021). Benefiting from advances in medical technology and the popularity of endoscopy, more and more gastric cancer is diagnosed at a relatively early stage. pT2N0M0 gastric cancer is defined as tumors infiltrating the muscularis propria without regional lymph node metastasis or distant metastasis based on the $8^{\text {th }}$ edition of the AJCC TNM staging system for gastric cancer (Amin et al., 2017; Brierley et al., 2017).

Surgery is the only potential chance of cure for gastric cancer, but a certain percentage of patients relapse after curative surgery, which leads to a poor prognosis. Adjuvant chemotherapy (ACT) or chemoradiotherapy has been demonstrated to be beneficial in numerous clinical trials worldwide (Macdonald et al., 2001; Sasako et al., 2011; Noh et al., 2014; Park et al., 2015). Nevertheless, these trials did not report whether patients with less advanced disease would benefit from adjuvant therapy. There are few studies on patients with pT2N0M0 gastric cancer.

Consensus guidelines provide disparate recommendations. Based on the National Comprehensive Cancer Network (NCCN) Guidelines (version 1.2021, Gastric Cancer), options for pT2N0M0 gastric cancer patients after D2 lymph node dissection include surveillance or ACT. Patients with poorly differentiated or high-grade cancer, lymphovascular invasion, neural invasion or aged $<50$ years are candidates for ACT (National and Comprehensive, 2020). Meanwhile, observation without adjuvant therapy after curative resection is recommended for stage I (including T2N0M0) gastric cancer according to the Japanese Gastric Cancer Treatment Guidelines 2018 ( $5^{\text {th }}$ edition) (Japanese Gastric Cancer A, 2020). ACT may decrease the risk of metastasis in high-risk pT2N0M0 patients, such as those aged $<40$ years or with high-grade or poorly differentiated tumor and nervous, lymphovascular invasion, based on the Chinese Society of Clinical Oncology (CSCO) Guidelines (version 1.2021, Gastric Cancer); however, it is unclear whether there is survival benefit of ACT for stage I gastric cancer ((Wang et al., 2021)).

Based on the 8th edition of the TNM staging system of gastric cancer, pT2N0M0 gastric cancer belongs to stage IB, which has good prognosis, with 5-year survival rate of approximately 80-90\% after curative surgery ((He et al., 2018; Ji et al., 2018)). The recurrence rates for pT2N0M0 gastric cancer after resection range from 3 to 9\% (Jin et al., 2015; Park et al., 2016). Considering the number of patients with stage I gastric cancer is increasing, several retrospective studies focused on the role of ACT in patients with pT2N0M0 gastric cancer and evaluated the high-risk factors of relapse and death; however, they reported diverse opinions on the effect of ACT on pT2N0M0 gastric cancer.
Since it was an open question whether ACT would benefit patients with pT2N0M0 gastric cancer, we aimed to determine the effect of ACT after curative resection in this study.

\section{MATERIALS AND METHODS}

\section{Patients}

All patients who underwent radical resection with D2 lymphadenectomy for primary gastric cancer and were ultimately diagnosed with pT2N0M0 gastric cancer based on the 8th edition of the AJCC TNM staging system for gastric cancer at Ruijin Hospital, Shanghai Jiao Tong University School of Medicine between January 2012 and May 2016 were reviewed. Patients with less than 16 harvested lymph nodes, other primary malignancies, prior gastric surgery, R1 or R2 surgical margins, age over 80 years, with postoperative complications; who died within 30 days of surgery; who were lost to follow-up and who received preoperative treatment were excluded. All surgeons had experience doing gastric surgery ( $>100$ procedures per year) and the standard operating procedures were based on the principles of surgery of CSCO Guidelines. Finally, a total of 307 patients were included in this study (Figure 1). This study was approved by the Ruijin Hospital Ethics Committee, Shanghai Jiao Tong University School of Medicine, China (No. 2018-151).

\section{Evaluation of Clinical Pathological Variables}

Clinical pathological characteristics, including age, sex, tumor size, location, Borrmann type, differentiation, histopathology, invasion depth, number of examined lymph nodes, lymphovascular invasion, perineural invasion, resection patterns and treatment regimen after surgery were analyzed. Age was converted to categorical variable, and the cutoff value (40 years) was decided based on the high-risk factors according to the CSCO guidelines (Wang et al., 2019a). Tumor location was classed as the upper, middle, or lower third of the stomach. Tumor histopathology was reviewed based on the WHO classification of the digestive system tumors, $5^{\text {th }}$ edition (WHO Classification of Tumours Editorial Board 2019). Histological type was divided into two groups: differentiated type (including well differentiated and moderately differentiated tubular adenocarcinoma) and undifferentiated type (including mucinous adenocarcinoma, signet ring cell carcinoma and poorly differentiated adenocarcinoma). The tumor invasion depth was divided into the superficial muscularis propria (sMP) layer and the deep muscularis propria (dMP) layer according to pathological examination (Sun et al., 2009). This category was based on the type of muscularis propria fibers; the transverse and longitudinal muscle layers were classified as the sMP and dMP layers, respectively. Lymphovascular invasion was defined as malignant cells appearing in a vascular wall structure or tubular space lined by endothelial cells. Perineural invasion was diagnosed when tumor cells were present in the perineural space of nerves. Total or subtotal gastrectomy was conducted 


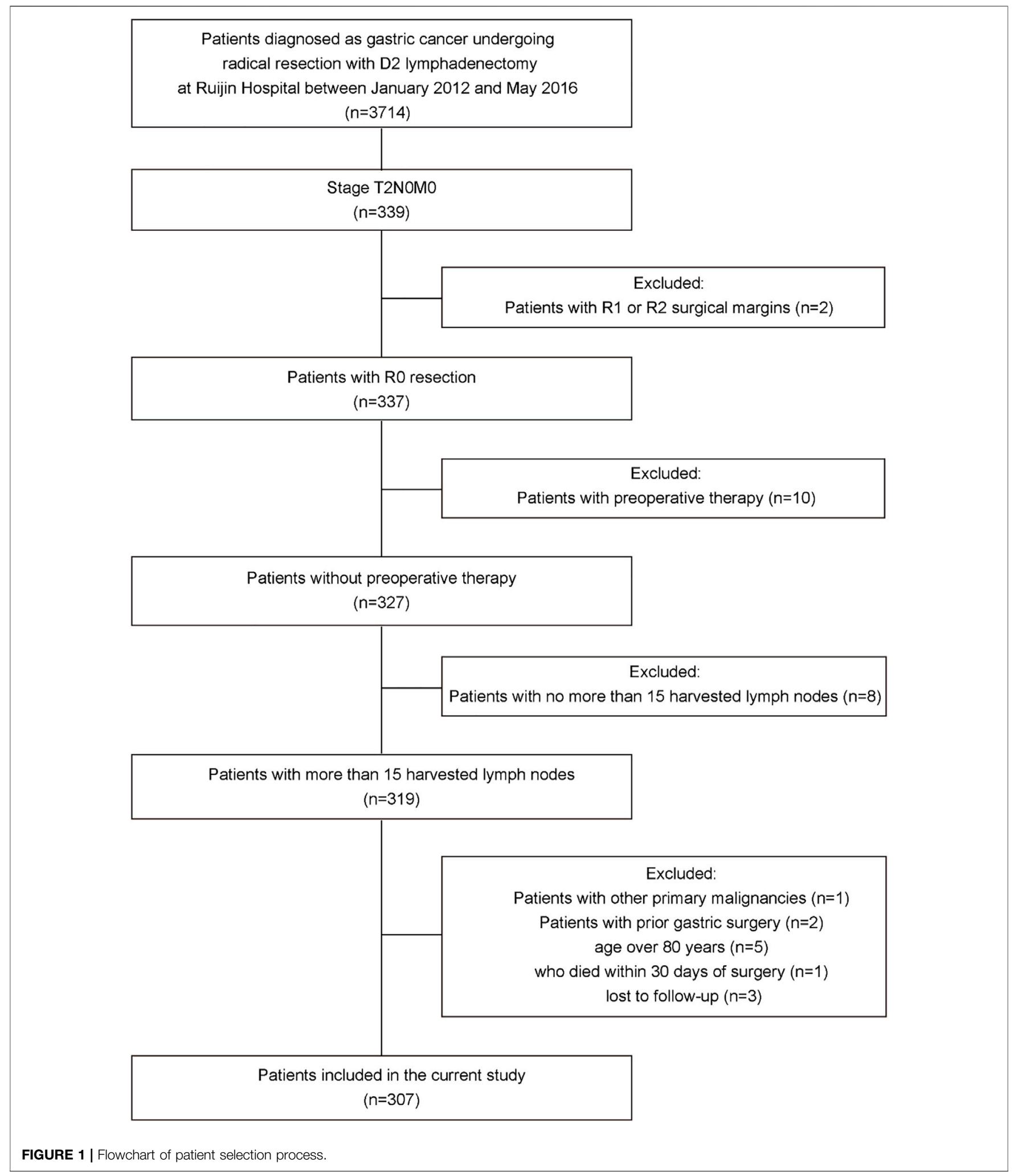

based on the tumor location. Two independent, experienced pathologists reviewed hematoxylin-eosin (H\&E)-stained slides from each case. If the diagnosis of the two pathologists was inconsistent, a third pathologist was needed.

\section{Treatment After Surgery}

All patients received postoperative examinations within 3-4 weeks after surgery and patients who received ACT start therapy within 4-6 weeks after surgery. All patients 
TABLE 1 | Clinical pathological characteristics of patients with pT2NOMO gastric cancer.

\begin{tabular}{|c|c|c|c|c|}
\hline Variables & Total $(n=307)$ & $\begin{array}{l}\text { With high-risk factors } \\
\qquad(n=216)\end{array}$ & $\begin{array}{l}\text { Without high-risk factors } \\
\qquad(n=91)\end{array}$ & $p$ Value \\
\hline Age (years) & & & & $0.022^{*}$ \\
\hline Median (IQRs) & $63(56,71)$ & $63(55,71)$ & $64(60,71)$ & \\
\hline$<40$ & 12 (3.9) & $12(5.6)$ & 0 & \\
\hline$\geq 40$ & $295(96.1)$ & $204(94.4)$ & $91(100)$ & \\
\hline Sex & & & & $0.006^{\star}$ \\
\hline Male & $216(70.4)$ & $142(65.7)$ & 74 (81.3) & \\
\hline Female & $91(29.6)$ & 74 (34.3) & $17(18.7)$ & \\
\hline Location & & & & 0.913 \\
\hline Upper & 55 (17.9) & 40 (18.5) & 15 (16.5) & \\
\hline Middle & 46 (15.0) & $32(14.8)$ & $14(15.4)$ & \\
\hline Lower & $206(67.1)$ & $144(66.7)$ & $62(68.1)$ & \\
\hline Size (cm) & & & & 0.989 \\
\hline$\leq 2.5$ & $155(50.5)$ & $109(50.5)$ & $46(50.5)$ & \\
\hline$>2.5$ & $152(49.5)$ & $107(49.5)$ & 45 (49.5) & \\
\hline Borrmann & & & & 0.661 \\
\hline | & $39(12.7)$ & 26 (12.0) & 13 (14.3) & \\
\hline$\|$ & $111(36.2)$ & $76(35.2)$ & 35 (38.5) & \\
\hline III & $157(51.1)$ & $114(52.8)$ & $43(47.3)$ & \\
\hline Differentiation & & & & $<0.001^{*}$ \\
\hline Differentiated & 96 (31.3) & $5(2.3)$ & $91(100)$ & \\
\hline Undifferentiated & $211(68.7)$ & $211(97.7)$ & 0 & \\
\hline Histopathology & & & & $<0.001^{\star}$ \\
\hline Tub & $96(31.3)$ & 5 (2.3) & $91(100)$ & \\
\hline Por & $160(52.1)$ & $160(74.1)$ & 0 & \\
\hline Sig & $32(10.4)$ & $32(14.8)$ & 0 & \\
\hline Muc & $19(6.2)$ & $19(8.8)$ & 0 & \\
\hline Depth & & & & 0.356 \\
\hline sMP & $163(53.1)$ & $111(51.4)$ & $52(57.1)$ & \\
\hline $\mathrm{dMP}$ & $144(46.9)$ & $105(48.6)$ & $39(42.9)$ & \\
\hline Examined LNs (Median (IQRs)) & $22(18,29)$ & $21(18,29)$ & $23(18,28)$ & 0.425 \\
\hline $\mathrm{PNI}$ & $23(7.5)$ & $23(10.6)$ & 0 & $0.001^{\star}$ \\
\hline LVI & $33(10.7)$ & $33(15.3)$ & 0 & $<0.001^{*}$ \\
\hline Gastrectomy & & & & 0.652 \\
\hline Distal & $224(73.0)$ & $156(72.2)$ & $68(74.7)$ & \\
\hline Total & $83(27.0)$ & $60(27.8)$ & $23(25.3)$ & \\
\hline Postoperative treatment & & & & $0.035^{\star}$ \\
\hline ACT & $196(63.8)$ & $146(67.6)$ & $50(54.9)$ & \\
\hline SA & $111(36.2)$ & $70(32.4)$ & $41(45.1)$ & \\
\hline ACT type & & & & $0.018^{\star}$ \\
\hline Monotherapy & $130(66.3)$ & $90(61.6)$ & $40(80.0)$ & \\
\hline Dual drug & $66(33.7)$ & $56(38.4)$ & $10(20.0)$ & \\
\hline
\end{tabular}

$\mathrm{p}<0.05$ was considered statistically significant.

High-risk factors including patients aged <40 years or with high-grade or poorly differentiated tumor and nervous, lymphovascular invasion, according to the CSCO Guidelines (version 1.2018, Gastric Cancer); Tub, tubular adenocarcinoma; Por, poorly differentiated adenocarcinoma; Sig, signet ring cell carcinoma; Muc, mucinous adenocarcinoma; sMP, superficial muscularis propria layer; dMP, deep muscularis propria layer; LNs, Iymph nodes; IQRs, interquartile ranges; PNI, perineural invasion; LVI, Iymphovascular invasion; ACT, adjuvant chemotherapy; SA, surgery alone.

included in my study was with adequate organ function for chemotherapy and PS $0-1$. Decisions to administer ACT to patients with pT2N0M0 gastric cancer were based on the preference of surgeons or oncologists. Some doctors approve the Japanese guidelines, thereby they do not recommend postoperative chemotherapy for pT2N0M0 gastric cancer patients; some doctors follow the Chinese guidelines, so they recommend postoperative chemotherapy for patients with high-risk factors based on the CSCO guidelines. Patients with younger age, undifferentiated tumor, perineural or lymphovascular invasion were more likely to receive dual-drug regimen. Patients were given S-1 as monotherapy, while the dual-drug regimen included XELOX or SOX. S-1 was given as follows: $40 \mathrm{mg} / \mathrm{m} 2$ p. o. b. i.d. day 1-day 14, Q3W for 1 year (Sasako et al., 2011). XELOX was given as six 3 -week cycles of capecitabine $(1,000 \mathrm{mg} / \mathrm{m} 2 \mathrm{p}$. o. b. i.d. days 1-14) plus oxaliplatin $(130 \mathrm{mg} / \mathrm{m} 2$ iv. day 1$)$ (Noh et al., 2014). SOX was given as six 3-week cycles of S-1 $(40 \mathrm{mg} / \mathrm{m} 2$ p. o. b. i.d. days $1-14)$ plus oxaliplatin $(130 \mathrm{mg} / \mathrm{m} 2$ iv. day 1) (Park et al., 2021). Adverse events were assessed by 
A

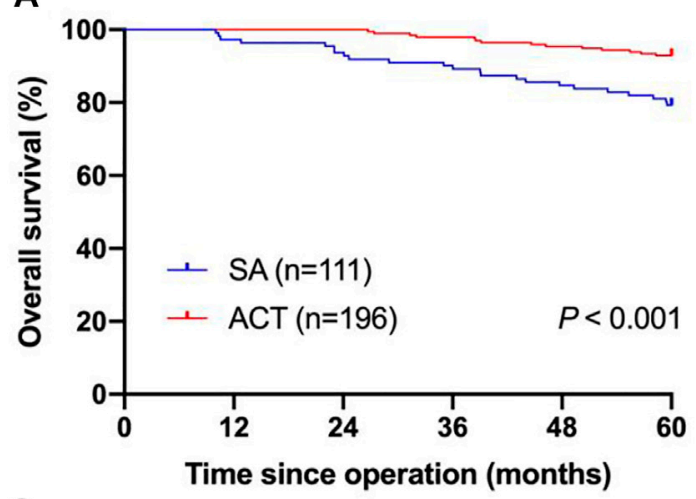

C

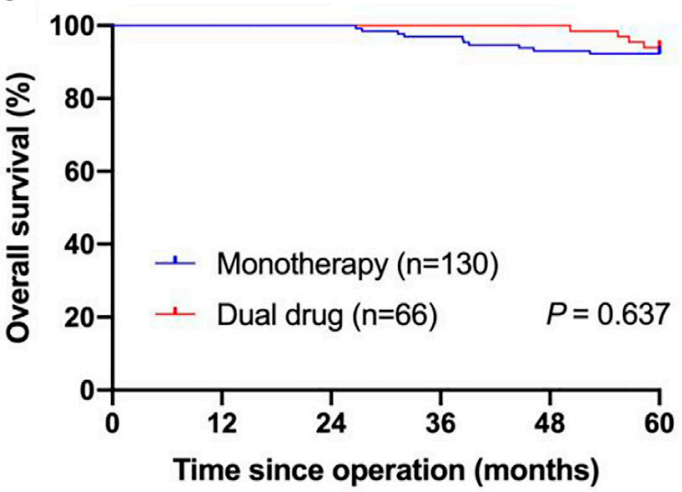

E

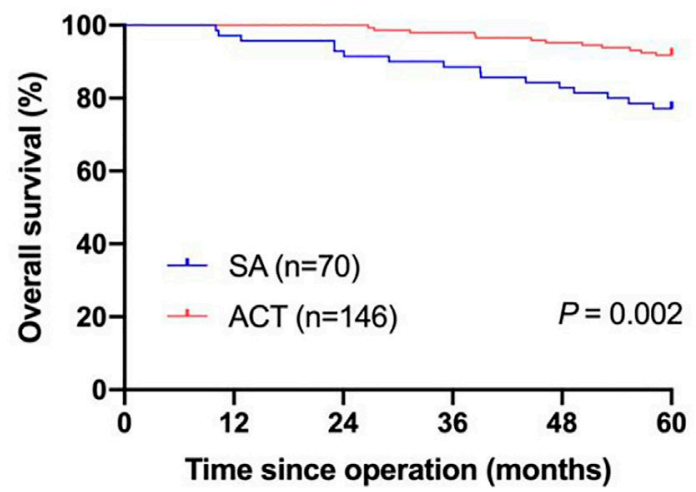

B
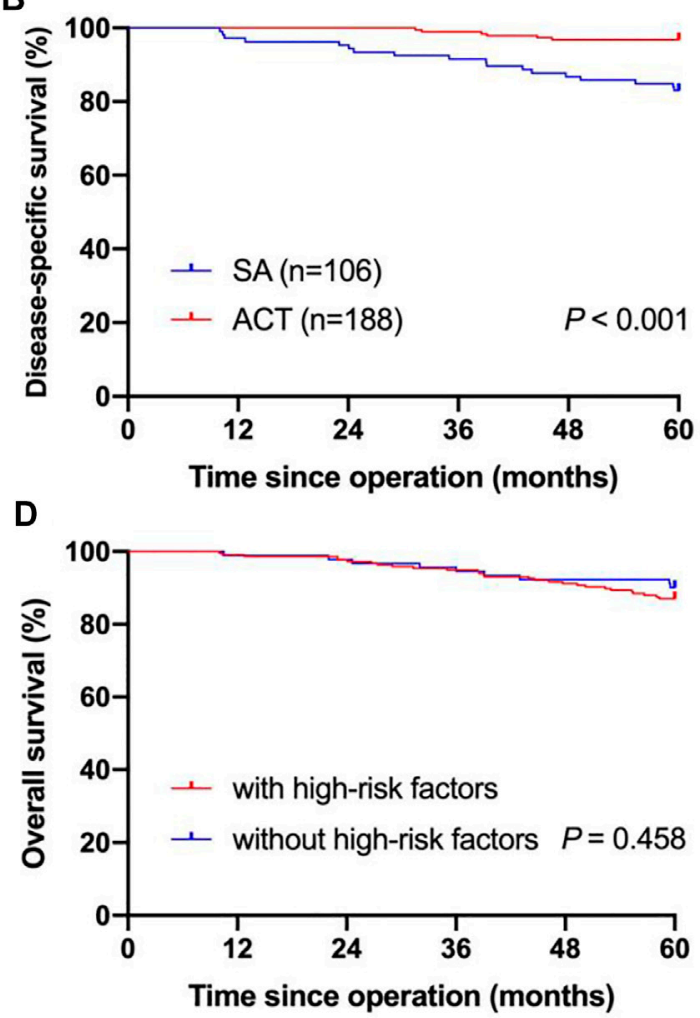

$\mathbf{F}$

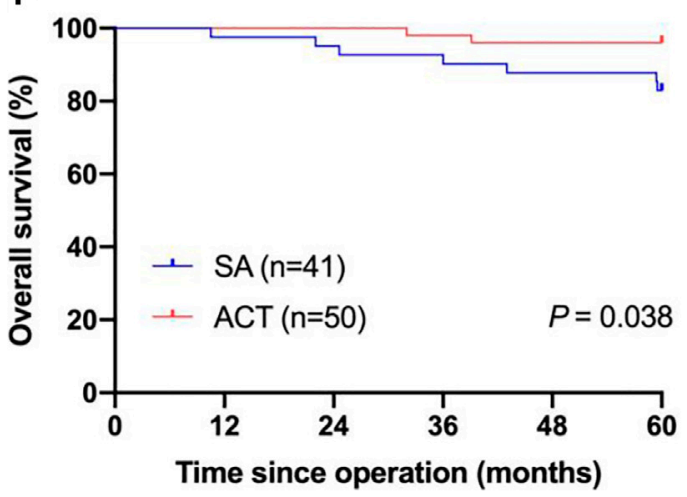

FIGURE 2 | Kaplan-Meier curves for survival of pT2NOMO gastric cancer patients. (A) Kaplan-Meier curves of pT2NOMO gastric cancer patients underwent SA and patients receiving ACT in the OS analysis; (B) Kaplan-Meier curves of pT2NOMO gastric cancer patients underwent SA and patients receiving ACT in the DSS analysis; (C) Kaplan-Meier curves of pT2NOMO gastric cancer patients who received monotherapy and patients who received the dual-drug regimen in the OS analysis; (D) Kaplan-Meier curves of pT2NOMO gastric cancer patients with high-risk factors and patients without high-risk factors in the OS analysis; (E) Kaplan-Meier curves of pT2NOMO gastric cancer patients with high-risk factors who underwent SA and who received ACT in the OS analysis; (F) Kaplan-Meier curves of pT2NOMO gastric cancer patients without high-risk factors who underwent SA and who received ACT in the OS analysis; SA, surgery alone; ACT, adjuvant chemotherapy; OS, overall survival; DSS, disease-specific survival.

the Common Terminology Criteria for Adverse Events (version 5.0). Dose reduction or interruption were allowed if patients had adverse events of grade 3 or 4 . Patients underwent surgery alone accepted no anticancer therapy until recurrence. When cancer relapse was observed, firstline treatment was administered.

\section{Follow-Up}

Outpatient follow-up was conducted every 3 months in the first 2 years and every 6 months for the next 3 years and included a physical examination, blood tests, and tumor markers. Chestabdomen-pelvis CT and endoscopy were performed every 6 months. Liver MRI, bone scans and PET were optional. The 
TABLE 2 | Univariate and Cox-regression of overall survival of patients with pT2NOMO gastric cancer.

\begin{tabular}{|c|c|c|c|c|c|}
\hline \multirow[t]{2}{*}{ Variables } & \multirow[t]{2}{*}{5 -year OS rate $(\%)$} & \multirow[t]{2}{*}{$p$ Value } & \multicolumn{3}{|c|}{ Cox-regression } \\
\hline & & & HR & $95 \% \mathrm{Cl}$ & $p$ Value \\
\hline Age (years) & & 0.665 & & & \\
\hline$<40$ & 91.7 & & & & \\
\hline$\geq 40$ & 87.8 & & & & \\
\hline Sex & & 0.126 & & & \\
\hline Male & 86.1 & & & & \\
\hline Female & 92.3 & & & & \\
\hline Location & & 0.773 & & & \\
\hline Upper & 85.5 & & & & \\
\hline Middle & 87.0 & & & & \\
\hline Lower & 88.8 & & & & \\
\hline Size (cm) & & 0.399 & & & \\
\hline$\leq 2.5$ & 86.5 & & & & \\
\hline$>2.5$ & 89.5 & & & & \\
\hline Borrmann & & 0.627 & & & \\
\hline । & 89.7 & & & & \\
\hline$\|$ & 85.6 & & & & \\
\hline III & 89.2 & & & & \\
\hline Differentiation & & 0.339 & & & \\
\hline Differentiated & 90.6 & & & & \\
\hline Undifferentiated & 86.7 & & & & \\
\hline Histopathology & & 0.776 & & & \\
\hline Tub & 90.6 & & & & \\
\hline Por & 86.3 & & & & \\
\hline Sig & 87.5 & & & & \\
\hline Muc & 89.5 & & & & \\
\hline Depth & & 0.327 & & & \\
\hline sMP & 89.6 & & & & \\
\hline $\mathrm{dMP}$ & 86.1 & & & & \\
\hline Examined LNs & & 0.556 & & & \\
\hline LVI & & 0.273 & & & \\
\hline Negative & 87.2 & & & & \\
\hline Positive & 93.9 & & & & \\
\hline PNI & & 0.166 & & & \\
\hline Negative & 88.7 & & & & \\
\hline Positive & 78.3 & & & & \\
\hline Gastrectomy & & $0.001^{\star}$ & & & \\
\hline Distal & 91.5 & & & & \\
\hline Total & 78.3 & & 2.445 & $1.279-4.675$ & $0.007^{\star}$ \\
\hline Postoperative treatment & & $<0.001^{\star}$ & & & \\
\hline SA & 79.3 & & 2.913 & $1.494-5.682$ & $0.002^{*}$ \\
\hline ACT & 92.9 & & & & \\
\hline ACT type & & 0.637 & & & \\
\hline Monotherapy & 92.3 & & & & \\
\hline Dual drug & 93.9 & & & & \\
\hline
\end{tabular}

${ }^{*} \mathrm{p}<0.05$ was considered statistically significant.

Tub, tubular adenocarcinoma; Por, poorly differentiated adenocarcinoma; Sig, signet ring cell carcinoma; Muc, mucinous adenocarcinoma; sMP, superficial muscularis propria layer; dMP, deep muscularis propria layer; LNs, lymph nodes; PNI, perineural invasion; LVI, lymphovascular invasion; ACT, adjuvant chemotherapy; SA, surgery alone; HR, hazard ratio; 95\% Cl, $95 \%$ confidence interval.

follow-up lasted at least 5 years after surgery or until censoring date or death.

\section{Statistical Analysis}

Continuous variable is shown as median with interquartile ranges (IQRs), and categorical variable is presented as number with proportions. Categorical variable was analyzed using Fisher's exact test or chi square test. DSS was defined as the time of surgery to death from gastric cancer. The 5-year OS and DSS rates were calculated using the Kaplan-Meier curve, and differences were analyzed by the log-rank test. Independent predictors of survival were found by Cox-regression survival analysis. Hazard ratio $(\mathrm{HR})>1$ was related to a higher hazard of death. A $p$ value $<$ 0.05 was considered as statistically significant. SPSS version 22.0 for Windows (IBM Corporation, Armonk, NY, United States) was used for statistical analysis.

\section{RESULTS}

\section{Clinical Pathological Features of Patients With pT2NOMO Gastric Cancer}

A total of 307 patients with pT2N0M0 gastric cancer were enrolled in this study. The clinical pathological characteristics 
are shown in Table 1. The age ranged from 29 to 80 years, with a median age of 63 years. Most of patients were male $(n=216$, $70.4 \%$ ). The median tumor size was $2.5 \mathrm{~cm}$. Size was converted to categorical variable, and the cutoff value was median size. Tumors were more likely located in the lower $1 / 3$ of the stomach $(n=206$, $67.1 \%)$ and presented as the Borrmann III type ( $n=151,51.1 \%)$ and undifferentiated type $(\mathrm{n}=211,68.7 \%)$. The median number of harvested lymph nodes was 22 with a range from 16 to 68 . Twenty-three patients had perineural invasion and 33 patients had lymphovascular invasion. $63.8 \%$ of patients received ACT, 130 patients received monotherapy and 66 patients were given dual drug treatment.

\section{Long-Term Outcomes and Effect of ACT on Prognosis in pT2NOMO Gastric Cancer Patients}

As of May 2021, the median follow-up was 73.1 months, ranging from 10 to 112.9 months. In our study, 5-year OS rate of all patients was $87.9 \%$. Kaplan-Meier survival analysis showed that the 5-year OS rate was higher in patients who received ACT (92.9\%) compared with those who underwent surgery alone (79.3\%, $p<0.001$, Figure 2A). In the Cox-regression analysis, independent predictors of decreased OS were surgery alone (HR $=2.913,95 \%$ CI: $1.494-5.682, p=0.002)$ and total gastrectomy (HR $=2.445,95 \%$ CI: 1.279-4.675, $p=0.007$, Table 2).

The 5-year DSS rate of enrolled patients was $91.8 \%$ when excluding 13 patients who did not die from gastric cancer. Supplementary Table $\mathbf{S 1}$ shows the clinical pathological characteristics of patients enrolled in the DSS analysis. Patients receiving ACT showed a better 5-year DSS rate (96.8 vs. $83.0 \%$, $p<0.001)$ than patients underwent surgery alone with significant difference (Figure 2B). In the Cox-regression analysis, surgery alone $(\mathrm{HR}=5.052,95 \% \mathrm{CI}: 1.993-12.809, p=0.001)$ and total gastrectomy $(\mathrm{HR}=2.820,95 \% \mathrm{CI}: 1.256-6.329, p=0.012)$ were independently associated with decreased OS (Supplementary Table S2). Supplementary Table S4 shows the dominant recurrence sites in patients who died of gastric cancer relapse.

\section{Effect of the ACT Regimen on Prognosis in pT2NOMO Gastric Cancer}

Of 196 patients received ACT, 130 patients received monotherapy, and 66 patients received dual-drug chemotherapy. Supplementary Table S3 shows the clinical pathological variables of patients who were given different chemotherapy regimens. The clinical pathological characteristics between the two groups were comparable, except for age, differentiation, lymphovascular invasion and perineural invasion. Most patients with lymphovascular invasion or perineural invasion received dual-drug chemotherapy, and the 5-year OS rate of the dual-drug subgroup reached $93.9 \%$, while the monotherapy subgroup had a relatively poor prognosis, without a significant difference (92.3\%, $p=0.637$, Figure $2 \mathrm{C}$ ).

Grade 5 adverse events did not occur. The main grade 3 or 4 adverse events were anemia (9.2\%), anorexia (6.9\%) diarrhea
(4.6\%) in the monotherapy group and neutropenia (15.2\%), peripheral neuropathy $(12.1 \%)$, anorexia $(7.6 \%)$ and anemia $(4.5 \%)$ in the dual-drug regimen group.

\section{Stratification by High-Risk Factors According to the CSCO Guidelines}

According to the CSCO guidelines, high-risk factors include patients aged <40 years or with high-grade or poorly differentiated tumor and nervous, lymphovascular invasion. Seventy-three patients had high-risk factors and thirty-four patients did not have high-risk factors. The clinical pathological features of patients stratified by high-risk factors was showed in Table 1. The 5-year OS rate was lower in patients with high-risk factors (87.0\%) compared with those without highrisk factors $(90.1 \%)$, whereas the difference was not statistically significant ( $p=0.458$, Figure 2D).

In patients with high-risk factors, gastrectomy type and postoperative therapy were concerned with prognosis in the univariate analysis. In patients with high-risk factors, the 5-year OS rate of patients received ACT was significantly higher than that of patients underwent surgery alone $(91.8$ vs $77.1 \%, p=$ 0.002 , Figure 2E). In the Cox-regression analysis, surgery alone $(\mathrm{HR}=3.130,95 \% \mathrm{CI}: 1.480-6.620, p=0.003)$ and total gastrectomy $(\mathrm{HR}=3.303,95 \% \mathrm{CI}: 1.571-6.947, p=0.002)$ were independently associated with decreased OS (Table 3). In patients without high-risk factors, the 5-year OS rate of patients received ACT was also significantly higher than that of patients underwent surgery alone $(96.0$ vs $82.9 \%, p=0.038$, Figure 2F). Thus, ACT could not only increase the 5-year survival rate of patients with high-risk factors, but also increase the 5-year survival rate of patients without high-risk factors.

In patients without high-risk factors, 40 patients received monotherapy, 10 patients received dual-drug regimen. The 5year OS rate was $95.0 \%$ for the monotherapy subgroup and $100 \%$ for the dual-drug subgroup without significant difference $(p=$ 0.477 , Table 3). In patients with high risk factors, 90 patients received monotherapy, 56 patients received dual-drug regimen. The 5-year OS rate was $91.1 \%$ for the monotherapy subgroup and $92.9 \%$ for the dual-drug subgroup without significant difference $(p=0.664$, Table 3).

\section{DISCUSSION}

ACTS-GC trial (Sasako et al., 2011) demonstrated that patients with stage II/III gastric cancer could significantly benefit from adjuvant S-1. CLASSIC trial (Noh et al., 2014) also showed survival benefit of adjuvant XELOX for stage II/III gastric cancer patients. ARTIST II trial (Park et al., 2021) showed that adjuvant SOX was more effective than S-1 in patients with node positive, stage II/III gastric cancer. Exiting prospective randomized clinical trials demonstrating the benefit of ACT could not explain whether all gastric cancer patients (especially stage IB gastric cancer) would benefit from ACT. Although the prognosis of pT2N0M0 gastric cancer is 
TABLE 3 | Univariate and Cox-regression of overall survival of patients with pT2NOMO gastric cancer stratified by high-risk factors.

\begin{tabular}{|c|c|c|c|c|c|c|c|}
\hline \multirow[t]{3}{*}{ Variables } & \multicolumn{5}{|c|}{ With high-risk factors } & \multicolumn{2}{|c|}{ Without high-risk factors } \\
\hline & \multirow[t]{2}{*}{ 5-year OS rate } & \multirow[t]{2}{*}{$P$ value } & \multicolumn{3}{|c|}{ Cox-regression } & \multirow[t]{2}{*}{ 5-year OS rate } & \multirow[t]{2}{*}{$P$ value } \\
\hline & & & HR & $95 \% \mathrm{Cl}$ & $P$ value & & \\
\hline Age (years) & & 0.601 & & & & - & - \\
\hline$<40$ & $91.7 \%$ & & & & & & \\
\hline$\geq 40$ & $86.8 \%$ & & & & & & \\
\hline Sex & & 0.122 & & & & & 0.534 \\
\hline Male & $84.5 \%$ & & & & & $89.2 \%$ & \\
\hline Female & $91.9 \%$ & & & & & $94.1 \%$ & \\
\hline Location & & 0.479 & & & & & 0.797 \\
\hline Upper & $82.5 \%$ & & & & & $93.3 \%$ & \\
\hline Middle & $84.4 \%$ & & & & & $92.9 \%$ & \\
\hline Lower & $88.9 \%$ & & & & & $88.7 \%$ & \\
\hline Size (cm) & & 0.420 & & & & & 0.754 \\
\hline$\leq 2.5$ & $85.3 \%$ & & & & & $89.1 \%$ & \\
\hline$>2.5$ & $88.8 \%$ & & & & & $91.1 \%$ & \\
\hline Borrmann & & 0.654 & & & & & 0.311 \\
\hline | & $92.3 \%$ & & & & & $84.6 \%$ & \\
\hline$\|$ & $85.5 \%$ & & & & & $85.7 \%$ & \\
\hline III & $86.8 \%$ & & & & & $95.3 \%$ & \\
\hline Differentiation & & 0.399 & & & & - & - \\
\hline Differentiated & $100 \%$ & & & & & & \\
\hline Undifferentiated & $86.7 \%$ & & & & & & \\
\hline Histopathology & & 0.829 & & & & - & - \\
\hline Tub & $100 \%$ & & & & & & \\
\hline Por & $86.3 \%$ & & & & & & \\
\hline Sig & $87.5 \%$ & & & & & & \\
\hline Muc & $89.5 \%$ & & & & & & \\
\hline Depth & & 0.536 & & & & & 0.410 \\
\hline sMP & $88.3 \%$ & & & & & $92.3 \%$ & \\
\hline $\mathrm{dMP}$ & $85.7 \%$ & & & & & $87.2 \%$ & \\
\hline Examined LNs & & 0.927 & & & & & 0.210 \\
\hline LVI & & 0.211 & & & & - & - \\
\hline Negative & $85.8 \%$ & & & & & & \\
\hline Positive & $93.9 \%$ & & & & & & \\
\hline $\mathrm{PNI}$ & & 0.223 & & & & - & - \\
\hline Negative & $88.1 \%$ & & & & & & \\
\hline Positive & $78.3 \%$ & & & & & & \\
\hline Gastrectomy & & $0.001^{*}$ & & & & & 0.552 \\
\hline Distal & $91.7 \%$ & & & & & $91.2 \%$ & \\
\hline Total & $75.0 \%$ & & 3.303 & $1.571-6.947$ & $0.002^{\star}$ & $87.0 \%$ & \\
\hline Postoperative treatment & & $0.002^{*}$ & & & & & $0.038^{\star}$ \\
\hline SA & $77.1 \%$ & & 3.130 & $1.480-6.620$ & $0.003^{*}$ & $82.9 \%$ & \\
\hline ACT & $91.8 \%$ & & & & & $96.0 \%$ & \\
\hline ACT type & & 0.664 & & & & & 0.477 \\
\hline Monotherapy & $91.1 \%$ & & & & & $95.0 \%$ & \\
\hline Dual drug & $92.9 \%$ & & & & & $100 \%$ & \\
\hline
\end{tabular}

${ }^{*} \mathrm{p}<0.05$ was considered statistically significant.

High-risk factors including patients aged <40 years or with high-grade or poorly differentiated tumor and nervous, lymphovascular invasion, according to the CSCO Guidelines (version 1.2018, Gastric Cancer); Tub, tubular adenocarcinoma; Por, poorly differentiated adenocarcinoma; Sig, signet ring cell carcinoma; Muc, mucinous adenocarcinoma; sMP, superficial muscularis propria layer; dMP, deep muscularis propria layer; LNs, lymph nodes; PNI, perineural invasion; LVI, Iymphovascular invasion; ACT, adjuvant chemotherapy; SA, surgery alone; HR, hazard ratio; $95 \% \mathrm{Cl}$, 95\% confidence interval.

relatively good in general, postoperative relapse still occurs in some patients with various recurrence sites.

In the current study, we found a good prognosis of pT2N0M0 gastric cancer, with the 5 -year OS rate of $87.9 \%$ and 5 -year DSS rate of $91.8 \%$, similar to other studies ((In et al., 2016; Park et al., 2016)).

Some retrospective studies identified risk factors in stage I gastric cancer patients. The authors of a Korean study focusing on stage I gastric cancer reported that age, sex, stage IB, lymphatic vessel invasion, nerve invasion and a high serum carcinoembryonic antigen level, were independent prognostic factors (Caccialanza et al., 2016; Liu et al., 2016). A population-based study using the Surveillance, Epidemiology, and End Results (SEER) database demonstrated that older age, proximal tumor location, high tumor grade and large tumor size were independent factors of poor disease-related survival (Gold et al., 2013). Other studies found that several clinical pathological factors were significantly associated with a high risk of relapse and 
death in pT2N0M0 gastric cancer patients and suggested that patients with high-risk factors receive ACT. A further Chinese study identified the upper $1 / 3$ of the stomach, large tumor diameter, perineural and lymphovascular invasion as independent risk factors associated with decreased OS rates (Wang et al., 2018). Another study also reported that lymphatic vessel and nerve invasion and tumor size were independent risk factors (Caccialanza et al., 2016; Liu et al., 2016).

Our study found that total gastrectomy and surgery alone were independent risk factors for survival. Other studies also found many other risk factors associated with a poor prognosis. The main reason for this inconsistency was study heterogeneity, with differences in race, surgical practice and initial prognosis.

A single-center study from the CLASSIC trial (Caccialanza et al., 2016; Liu et al., 2016) found a marked loss in body composition parameters (muscle, visceral fat and subcutaneous fat) significantly predicted short disease-free survival and OS among patients who underwent gastrectomy. Malnutrition was considered as poor prognostic factor in cancer patients (Caccialanza et al., 2016; Liu et al., 2016). Fujiya demonstrated that persistent postoperative malnutrition was frequently observed in patients who underwent total gastrectomy (Fujiya et al., 2018). These studies might explain why patients who received total gastrectomy had poor prognoses in our study, although we could not evaluate the nutrition index.

Despite a lack of prospective studies that explored the benefit of ACT in less advanced gastric cancer, there were some retrospective studies exploring the effect of ACT such patients. Based on the 8th edition of the TNM staging system of gastric cancer, stage IB gastric cancer includes pT1N1M0 and pT2N0M0. Wang used the SEER database to explore the difference between T1N1M0 and T2N0M0 and found that patients with T2N0M0 gastric cancer may not benefit from adjuvant treatment (Wang et al., 2019b). Recently, Jin et al.(Jin et al., 2021) found that pT2N0 gastric cancer patients with non-signet ring cell carcinoma, tumor size $>3 \mathrm{~cm}$ and examined lymph nodes $\leq 15$ may be particularly appropriate candidates for ACT. In our study, there was no significant difference in OS between patients with signet ring cell carcinoma and patients with other histopathology type.

Since 1997, the retrieval of at least 15 lymph nodes has been recommended for adequate gastric cancer staging, and several studies have found that lymphadenectomy with $<15$ lymph nodes removed was an adverse independent prognostic factor for OS. A SEER study demonstrated that OS was dependent on the number of harvested lymph nodes; in patients with node-negative T1-2 gastric cancer, every additional 10 lymph nodes harvested increased the 5-year survival rate of $7.6 \%$ (Smith et al., 2005). Haejin found that their subgroup of T2N0M0 gastric cancer patients who underwent suboptimal lymphadenectomy benefitted from chemoradiotherapy rather than chemotherapy (Coburn et al., 2008; Du et al., 2011). Due to a lack of patients who received postoperative radiotherapy, the differences in radiotherapy and chemoradiotherapy roles could not be established in our study. Other studies failed to show the number of removed lymph nodes as an independent prognostic factor (Coburn et al., 2008; Du et al., 2011). One large population-based study demonstrated that surgery with adequate lymph node removing alone ( $\geq 15$ lymph nodes) predicted better prognosis compared with adjuvant therapy in patients with stage I or node-negative gastric cancer (Dudeja et al., 2012). Our study found that the number of harvested lymph nodes was not associated with prognosis, which may be related to excluding patients with fewer than 15 harvested lymph nodes.

Several studies on patients with pT2 gastric cancer focused on the invasion depth. Some studies have showed that pT2 gastric cancer patients showing invasion into dMP had a relatively poor prognosis than those only invasion sMP (Zhang et al., 2016; Park et al., 2021), while others reported no significant difference in the prognosis between the two groups (Son et al., 2007; Nakamura et al., 2019). In our study, the difference in the 5-year OS rate between the sMP and dMP subgroups was not significant (89.6 vs. $86.1 \%, p=0.327)$.

Regarding the therapy regimen, monotherapy and dual-drug therapy showed no significant difference. ACTS-GC trial and CLASSIC trial demonstrated that ACT with S-1 or XELOX was safe. In our study, the main grade 3 or 4 adverse events were neutropenia, peripheral neuropathy in the dual-drug group and anemia, anorexia in the monotherapy group. According to the ACTS-GC trial ((Sakuramoto et al., 2007)), the most common adverse events of grade 3 or grade 4 were anorexia (6.0\%), nausea (3.7\%), and diarrhea (3.1\%) in the S-1 group. According to the CLASSIC trial ((Bang et al., 2012)), the main grade 3 or 4 adverse events were neutropenia (22\%), thrombocytopenia (8\%), nausea $(8 \%)$, and vomiting $(7 \%)$ in the XELOX group. According the ARTIST II study ((Nagaraja et al., 2019; Li et al., 2020; Low et al., 2021; Nakazawa et al., 2021)), the most common adverse events of grade 3 or 4 were peripheral neuropathy (12\%), anemia (8\%) and anorexia (4\%) in the SOX group. The common dose-limiting toxicity of oxaliplatin is peripheral neuropathy, which affects $90 \%$ patients ((Kweekel et al., 2005)). The incidence of peripheral neuropathy is considered to be related to the prolonged use of oxaliplatin ((Baek et al., 2010)). Thus, we recommend monotherapy to prevent toxicity and discomfort. However, other studies, which aim to explore the role of ACT in stage IB gastric cancer, failed to analyse the difference between monotherapy and dual-drug therapy.

According to the CSCO guidelines, patients with pT2N0M0 gastric cancer with high-risk factors (age $<40$ years or with high-grade or poorly differentiated tumor and nervous, lymphovascular invasion) are recommended to receive ACT to reduce the risk of recurrence. Then, we divided patients with pT2N0M0 gastric cancer into two subgroups (with high-risk factors and without high-risk factors) and evaluated whether the effect of postoperative therapy was diverse. ACT indeed provided survival benefits to patients with high-risk factors, while patients without high-risk factors also benefitted from ACT, which was inconsistent with the CSCO guidelines. Regarding the therapy regimen, monotherapy and dual-drug therapy showed no significant difference; thus, considering possible adverse events, we recommend monotherapy regardless of high-risk factors. 
There were no studies exploring the role of ACT stratified by high-risk factors based on the CSCO guidelines.

Although patients with gastric cancer received ACT after radical gastrectomy, some patients still experienced relapse. Timely detection of recurrence, as well as identification of patients at high risk of relapse after surgery or completion of adjuvant therapy are major challenges in the treatment of gastric cancer. Drug resistance is the major factor of treatment failure and relapse and numerous studies aim to investigate the mechanisms of drug resistance ((Nagaraja et al., 2019; Li et al., 2020; Low et al., 2021; Nakazawa et al., 2021)). Over the past few decades, predictive biomarkers have received increasing attention in diagnosis, treatment, and prognosis of gastric cancer. Studies have found many predictive biomarkers for the precision treatment of gastric cancer (Petrillo and Smyth, 2020). In 2014, The Cancer Genome Atlas (Cancer Genome Atlas Resea, 2014) proposed a molecular classification of gastric cancer into 4 subtypes: chromosomal instability, Epstein-Barr virus positive, genomically stable and microsatellite instability (MSI). An et al.(An et al., 2012) found that in stage II/III gastric cancer, patients with microsatellite stable and MSI-low type significantly benefited from 5-FU-based ACT, while patients with MSIhigh type did not benefit from 5-FU-based ACT. Findings from the MAGIC trial (Smyth et al., 2017) showed that mismatch repair deficiency (dMMR) and MSI-high were associated with good prognosis in patients treated with surgery alone, whereas in gastric cancer patients treated with perioperative chemotherapy, $\mathrm{dMMR}$ and MSI-high were associated with worse prognosis. Post hoc analysis of CLASSIC trial (Choi et al., 2019) showed that MSI-high was independent prognostic factor and ACT significantly improved disease-free survival in MSS group while no benefit was found in the MSI-high group. MSI status could be used for precision treatment of gastric cancer in the future.

A prospective randomized trial comparing surgery alone with ACT in stage IB gastric cancer patients with at least one risk factor for recurrence (male sex, age $>65$ years, perineural and lymphovascular invasion) is now ongoing (ClinicalTrials.gov identifier NCT01917552), and this large-scale prospective trial is expected to compensate for previous research shortcomings and yield satisfactory results. Although the trial is based on the 6th edition of the AJCC staging system, it also includes pT2N0M0 gastric cancer based on the 8th edition of the AJCC staging system.

Nevertheless, there are several potential limitations in this study. The number of patients with pT2N0M0 gastric cancer was relatively small since it was a single-center study, the resultant effects may have been underestimated, and the results should be interpreted with caution. In addition, this was a retrospective study, and there were likely patient and tumor baseline characteristic imbalances between the treatment groups. Finally, the role of radiotherapy was not analysed due to a lack of patients who received postoperative radiotherapy.
Therefore, the conclusions of this study need to be verified by prospective study with a large sample size.

\section{CONCLUSION}

ACT was accompanied with higher 5-year OS and DSS rates of patients with pT2N0M0 gastric cancer. Patients with pT2N0M0 gastric cancer, regardless of high-risk factors based on the CSCO guidelines, might be considered candidates for ACT. In regard to the therapy regimen, monotherapy might be the optimal choice, considering the adverse events.

\section{DATA AVAILABILITY STATEMENT}

The original contributions presented in the study are included in the article/Supplementary Material, further inquiries can be directed to the corresponding authors.

\section{ETHICS STATEMENT}

The studies involving human participants were reviewed and approved by Ruijin Hospital Ethics Committee, Shanghai Jiao Tong University School of Medicine, China. The patients/ participants provided their written informed consent to participate in this study.

\section{AUTHOR CONTRIBUTIONS}

YM and XF designed the study, collected the patient data and drafted the paper. TF checked all the statistical calculations. MY, ZZ, TL, and ZZ participated in the design of the study and assisted in the collection of the data and edited the final paper. All authors read and approved the paper for publication.

\section{FUNDING}

This work was supported by the Multicenter Clinical Trial of Shanghai Jiao Tong University of Medicine (No. DLY201602) and the Medical Engineering Cross Research Fund of Shanghai Jiao Tong University (No. YG2021QN14).

\section{SUPPLEMENTARY MATERIAL}

The Supplementary Material for this article can be found online at: https://www.frontiersin.org/articles/10.3389/fphar.2022.845261/ full\#supplementary-material 


\section{REFERENCES}

Amin, M. B., Edge, S. B., Greene, F. L., and Brierley, J. D. (2017). AJCC Cancer Staging Manual. 8th ed. New York: Springer.

An, J. Y., Kim, H., Cheong, J. H., Hyung, W. J., Kim, H., and Noh, S. H. (2012). Microsatellite Instability in Sporadic Gastric Cancer: its Prognostic Role and Guidance for 5-FU Based Chemotherapy after R0 Resection. Int. J. Cancer 131 (2), 505-511. doi:10.1002/ijc.26399

Baek, K. K., Lee, J., Park, S. H., Park, J. O., Park, Y. S., Lim, H. Y., et al. (2010). Oxaliplatin-induced Chronic Peripheral Neurotoxicity: a Prospective Analysis in Patients with Colorectal Cancer. Cancer Res. Treat. 42 (4), 185-190. doi:10. 4143/crt.2010.42.4.185

Bang, Y. J., Kim, Y. W., Yang, H. K., Chung, H. C., Park, Y. K., Lee, K. H., et al. (2012). Adjuvant Capecitabine and Oxaliplatin for Gastric Cancer after D2 Gastrectomy (CLASSIC): a Phase 3 Open-Label, Randomised Controlled Trial. Lancet 379 (9813), 315-321. doi:10.1016/S01406736(11)61873-4

Brierley, J. D., Gospodarwicz, M. K., Wittekind, C., and Amin, M. B. (2017). TNM Classifcation of Maligant Tumours. 8th ed. Oxford: Wiley Black well.

Caccialanza, R., Pedrazzoli, P., Cereda, E., Gavazzi, C., Pinto, C., Paccagnella, A., et al. (2016). Nutritional Support in Cancer Patients: A Position Paper from the Italian Society of Medical Oncology (AIOM) and the Italian Society of Artificial Nutrition and Metabolism (SINPE). J. Cancer 7 (2), 131-135. doi:10.7150/jca. 13818

Cancer Genome Atlas Research N (2014). Comprehensive Molecular Characterization of Gastric Adenocarcinoma. Nature 513 (7517), 202-209. doi:10.1038/nature13480

Cao, W., Chen, H. D., Yu, Y. W., Li, N., and Chen, W. Q. (2021). Changing Profiles of Cancer burden Worldwide and in China: a Secondary Analysis of the Global Cancer Statistics 2020. Chin. Med. J. (Engl) 134 (7), 783-791. doi:10.1097/CM9. 0000000000001474

Choi, Y. Y., Kim, H., Shin, S. J., Kim, H. Y., Lee, J., Yang, H. K., et al. (2019). Microsatellite Instability and Programmed Cell Death-Ligand 1 Expression in Stage II/III Gastric Cancer: Post Hoc Analysis of the CLASSIC Randomized Controlled Study. Ann. Surg. 270 (2), 309-316. doi:10.1097/SLA. 0000000000002803

Coburn, N. G., Govindarajan, A., Law, C. H., Guller, U., Kiss, A., Ringash, J., et al. (2008). Stage-specific Effect of Adjuvant Therapy Following Gastric Cancer Resection: a Population-Based Analysis of 4,041 Patients. Ann. Surg. Oncol. 15 (2), 500-507. doi:10.1245/s10434-007-9640-0

Du, C., Zhou, Y., Huang, K., Zhao, G., Fu, H., and Shi, Y. (2011). Defining a HighRisk Subgroup of Pathological T2N0 Gastric Cancer by Prognostic Risk Stratification for Adjuvant Therapy. J. Gastrointest. Surg. 15 (12), 2153-2158. doi:10.1007/s11605-011-1684-6

Dudeja, V., Habermann, E. B., Abraham, A., Zhong, W., Parsons, H. M., Tseng, J. F., et al. (2012). Is There a Role for Surgery with Adequate Nodal Evaluation Alone in Gastric Adenocarcinoma? J. Gastrointest. Surg. 16 (2), 246-7. doi:10. 1007/s11605-011-1756-7

Fujiya, K., Kawamura, T., Omae, K., Makuuchi, R., Irino, T., Tokunaga, M., et al. (2018). Impact of Malnutrition after Gastrectomy for Gastric Cancer on LongTerm Survival. Ann. Surg. Oncol. 25 (4), 974-983. doi:10.1245/s10434-018-6342-8

Gold, J. S., Al Natour, R. H., Saund, M. S., Yoon, C., Sharma, A. M., Huang, Q., et al. (2013). Population-based Outcome of Stage IA-IIA Resected Gastric Adenocarcinoma: Who Should Get Adjuvant Treatment? Ann. Surg. Oncol. 20 (7), 2304-2310. doi:10.1245/s10434-012-2852-y

He, X., Wu, W., Lin, Z., Ding, Y., Si, J., and Sun, L. M. (2018). Validation of the American Joint Committee on Cancer (AJCC) 8th Edition Stage System for Gastric Cancer Patients: a Population-Based Analysis. Gastric Cancer 21 (3), 391-400. doi:10.1007/s10120-017-0770-1

In, H., Kantor, O., Sharpe, S. M., Baker, M. S., Talamonti, M. S., and Posner, M. C. (2016). Adjuvant Therapy Improves Survival for T2N0 Gastric Cancer Patients with Sub-optimal Lymphadenectomy. Ann. Surg. Oncol. 23 (6), 1956-1962. doi:10.1245/s10434-015-5075-1

Japanese Gastric Cancer A (2020). Japanese Gastric Cancer Treatment Guidelines 2018. 5th edition. Gastric Cancer. doi:10.1007/s10120-02001042-y
Ji, X., Bu, Z. D., Yan, Y., Li, Z. Y., Wu, A. W., Zhang, L. H., et al. (2018). The 8th Edition of the American Joint Committee on Cancer Tumor-NodeMetastasis Staging System for Gastric Cancer Is superior to the 7th Edition: Results from a Chinese Mono-Institutional Study of 1663 Patients. Gastric Cancer 21 (4), 643-652. doi:10.1007/s10120-0170779-5The 8th edition

Jin, L. X., Moses, L. E., Squires, M. H., 3rd, Poultsides, G. A., Votanopoulos, K., Weber, S. M., et al. (2015). Factors Associated with Recurrence and Survival in Lymph Node-Negative Gastric Adenocarcinoma: A 7-Institution Study of the US Gastric Cancer Collaborative. Ann. Surg. 262 (6), 999-1005. doi:10.1097/ SLA. 0000000000001084

Jin, P., Ji, X., Ma, S., Kang, W., Liu, H., Li, Y., et al. (2021). Adjuvant Chemotherapy Indications for Stage I Gastric Cancer Patients with Negative Lymph Node. Clin. Res. Hepatol. Gastroenterol. 45 (6), 101634. doi:10.1016/j.clinre.2021. 101634

Kweekel, D. M., Gelderblom, H., and Guchelaar, H. J. (2005). Pharmacology of Oxaliplatin and the Use of Pharmacogenomics to Individualize Therapy. Cancer Treat. Rev. 31 (2), 90-105. doi:10.1016/j.ctrv.2004. 12.006

Li, Z., Gao, X., Peng, X., May Chen, M. J., Li, Z., Wei, B., et al. (2020). Multi-omics Characterization of Molecular Features of Gastric Cancer Correlated with Response to Neoadjuvant Chemotherapy. Sci. Adv. 6, eaay4211. doi:10.1126/ sciadv.aay 4211

Liu, X., Qiu, H., Liu, J., Chen, S., Xu, D., Li, W., et al. (2016). A Novel Prognostic Score, Based on Preoperative Nutritional Status, Predicts Outcomes of Patients after Curative Resection for Gastric Cancer. J. Cancer 7 (14), 2148-2156. doi:10. $7150 /$ jca. 16455

Low, H. B., Wong, Z. L., Wu, B., Kong, L. R., Png, C. W., Cho, Y.-L., et al. (2021). DUSP16 Promotes Cancer Chemoresistance through Regulation of Mitochondria-Mediated Cell Death. Nat. Commun. 12 (1), 2284. doi:10. 1038/s41467-021-22638-7

Macdonald, J. S., Smalley, S. R., Benedetti, J., Hundahl, S. A., Estes, N. C., Stemmermann, G. N., et al. (2001). Chemoradiotherapy after Surgery Compared with Surgery Alone for Adenocarcinoma of the Stomach or Gastroesophageal junction. N. Engl. J. Med. 345 (10), 725-730. doi:10.1056/ NEJMoa010187

Nagaraja, A. K., Kikuchi, O., BassGenomics, A. J., and Therapies, T. (2019). Genomics and Targeted Therapies in Gastroesophageal Adenocarcinoma. Cancer Discov. 9 (12), 1656-1672. doi:10.1158/2159-8290.CD-19-0487

Nakamura, K., Ogimi, M., Tomioku, M., Hara, H., Nabeshima, K., and Nomura, E. (2019). Clinical Implication of Tumor-Invasive Status into the Muscularis Propria in T2 Gastric Cancer. In Vivo 33 (4), 1341-1346. doi:10.21873/ invivo. 11609

Nakazawa, N., Sohda, M., Ide, M., Shimoda, Y., Ubukata, Y., Kuriyama, K., et al. (2021). High L-type Amino Acid Transporter 1 Levels Are Associated with Chemotherapeutic Resistance in Gastric Cancer Patients. Oncology 99 (11), 732-739. doi:10.1159/000517371

National, Comprehensive, Cancer, Network (2020). National Comprehensive Cancer Network (NCCN) Guidelines. Version 3. Gastric Cancer.

Navashenaq, J. G., Shabgah, A. G., Banach, M., Jamialahmadi, T., Penson, P. E., Johnston, T. P., et al. (2021). The Interaction of Helicobacter pylori with Cancer Immunomodulatory Stromal Cells: New Insight into Gastric Cancer Pathogenesis. Semin. Cancer Biol. doi:10.1016/j.semcancer.2021. 09.014

Noh, S. H., Park, S. R., Yang, H. K., Chung, H. C., Chung, I. J., Kim, S. W., et al. (2014). Adjuvant Capecitabine Plus Oxaliplatin for Gastric Cancer after D2 Gastrectomy (CLASSIC): 5-year Follow-Up of an Open-Label, Randomised Phase 3 Trial. Lancet Oncol. 15 (12), 1389-1396. doi:10.1016/s1470-2045(14) 70473-5

Park, H. S., Kim, H. S., Beom, S. H., Rha, S. Y., Chung, H. C., Kim, J. H., et al. (2018). Marked Loss of Muscle, Visceral Fat, or Subcutaneous Fat after Gastrectomy Predicts Poor Survival in Advanced Gastric Cancer: Single-Center Study from the CLASSIC Trial. Ann. Surg. Oncol. 25 (11), 3222-3230. doi:10.1245/s10434018-6624-1

Park, J. H., Ryu, M. H., Kim, H. J., Ryoo, B. Y., Yoo, C., Park, I., et al. (2016). Risk Factors for Selection of Patients at High Risk of Recurrence or Death after Complete Surgical Resection in Stage I Gastric Cancer. Gastric Cancer 19 (1), 226-233. doi:10.1007/s10120-015-0464-5 
Park, S. H., Lim, D. H., Sohn, T. S., Lee, J., Zang, D. Y., Kim, S. T., et al. (2021). A Randomized Phase III Trial Comparing Adjuvant Single-Agent S1, S-1 with Oxaliplatin, and Postoperative Chemoradiation with S-1 and Oxaliplatin in Patients with Node-Positive Gastric Cancer after D2 Resection: the ARTIST 2 Trial 弥. Ann. Oncol. 32 (3), 368-374. doi:10. 1016/j.annonc.2020.11.017

Park, S. H., Sohn, T. S., Lee, J., Lim, D. H., Hong, M. E., Kim, K. M., et al. (2015). Phase III Trial to Compare Adjuvant Chemotherapy with Capecitabine and Cisplatin versus Concurrent Chemoradiotherapy in Gastric Cancer: Final Report of the Adjuvant Chemoradiotherapy in Stomach Tumors Trial, Including Survival and Subset Analyses. J. Clin. Oncol. 33 (28), 3130-3136. doi:10.1200/JCO.2014.58.3930

Petrillo, A., and Smyth, E. C. (2020). Biomarkers for Precision Treatment in Gastric Cancer. Visc. Med. 36 (5), 364-372. doi:10.1159/000510489

Sakuramoto, S., Sasako, M., Yamaguchi, T., Kinoshita, T., Fujii, M., Nashimoto, A., et al. (2007). Adjuvant Chemotherapy for Gastric Cancer with S-1, an Oral Fluoropyrimidine. N. Engl. J. Med. 357 (18), 1810-1820. doi:10.1056/ NEJMoa072252

Sasako, M., Sakuramoto, S., Katai, H., Kinoshita, T., Furukawa, H., Yamaguchi, T., et al. (2011). Five-year Outcomes of a Randomized Phase III Trial Comparing Adjuvant Chemotherapy with S-1 versus Surgery Alone in Stage II or III Gastric Cancer. J. Clin. Oncol. 29 (33), 4387-4393. doi:10.1200/JCO.2011.36.5908

Smith, D. D., Schwarz, R. R., and Schwarz, R. E. (2005). Impact of Total Lymph Node Count on Staging and Survival after Gastrectomy for Gastric Cancer: Data from a Large US-Population Database. J. Clin. Oncol. 23 (28), 7114-7124. doi:10.1200/JCO.2005.14.621

Smyth, E. C., Wotherspoon, A., Peckitt, C., Gonzalez, D., Hulkki-Wilson, S., Eltahir, Z., et al. (2017). Mismatch Repair Deficiency, Microsatellite Instability, and Survival: An Exploratory Analysis of the Medical Research Council Adjuvant Gastric Infusional Chemotherapy (MAGIC) Trial. JAMA Oncol. 3 (9), 1197-1203. doi:10.1001/jamaoncol.2016.6762

Son, H. J., Son, H., Myung, W., Yoo, H. S., Park, S. H., Song, S. Y., et al. (2007). Prognostic Indicators of Gastric Carcinoma Confined to the Muscularis Propria. Histopathology 51 (1), 105-110. doi:10.1111/j.1365-2559.2007.02725.x

Sukri, A., Hanafiah, A., Mohamad Zin, N., and Kosai, N. R. (2020). Epidemiology and Role of Helicobacter pylori Virulence Factors in Gastric Cancer Carcinogenesis. APMIS 128 (2), 150-161. doi:10.1111/apm.13034

Sun, M., Ding, H., Zhu, Z., Wang, S., Gu, X., Xia, L., et al. (2021). Identifying Optimal Surgical Intervention-Based Chemotherapy for Gastric Cancer Patients with Liver Metastases. Front. Oncol. 11, 675870. doi:10.3389/fonc. 2021.675870

Sun, Z., Zhu, G. L., Lu, C., Guo, P. T., Huang, B. J., Li, K., et al. (2009). A Novel Subclassification of pT2 Gastric Cancers According to the Depth of Muscularis Propria Invasion: Superficial Muscularis Propria versus Deep Muscularis Propria/subserosa. Ann. Surg. 249 (5), 768-775. doi:10.1097/SLA. 0b013e3181a3df77
Varon, C., Azzi-Martin, L., Khalid, S., Seeneevassen, L., Menard, A., and Spuul, P. (2021). Helicobacters and Cancer, Not Only Gastric Cancer? Semin. Cancer Biol. doi:10.1016/j.semcancer.2021.08.007

Wang, F. H., Shen, L., Li, J., Zhou, Z. W., Liang, H., Zhang, X. T., et al. (2019). The Chinese Society of Clinical Oncology (CSCO): Clinical Guidelines for the Diagnosis and Treatment of Gastric Cancer. Cancer Commun. (Lond) 39 (1), 10. doi:10.1186/s40880-019-0349-9

Wang, F. H., Zhang, X. T., Li, Y. F., Tang, L., Qu, X. J., Ying, J. E., et al. (2021). The Chinese Society of Clinical Oncology (CSCO): Clinical Guidelines for the Diagnosis and Treatment of Gastric Cancer, 2021. Cancer Commun. (Lond) 41 (8), 747-795. doi:10.1002/cac2.12193

Wang, Y., Zhang, J., Guo, S., Dong, Z., Meng, X., Zheng, G., et al. (2019). Implication of Lymph Node Staging in Migration and Different Treatment Strategies for Stage T2N0M0 and T1N1M0 Resected Gastric Cancer: a SEER Population Analysis. Clin. Transl Oncol. 21 (11), 1499-1509. doi:10.1007/ s12094-019-02078-y

Wang, Z., Yan, J., Hu, W., Zhang, J., and Huo, B. (2018). Adjuvant Chemotherapy provided Survival Benefit for Stage T2N0 Gastric Cancer with High-Risk Factors. Neoplasma 65 (4), 592-598. doi:10. 4149/neo_2018_170620N436

WHO Classification of Tumours Editorial Board (2019). "WHO Classification of Tumours," in digestive system tumours. 5th edn. Lyon: Interational Agency for Research on Cancer.

Zeng, Y., and Jin, R. U. (2021). Molecular Pathogenesis, Targeted Therapies, and Future Perspectives for Gastric Cancer. Semin. Cancer Biol. doi:10.1016/j. semcancer.2021.12.004

Zhang, W. H., He, D., Chen, D. N., Li, T. T., Chen, X. Z., Yang, K., et al. (2016). Comparison between Superficial Muscularis Propria and Deep Muscularis Propria Infiltration in Gastric Cancer Patients: A Retrospective Cohort Study. Medicine (Baltimore) 95 (29), e4165. doi:10.1097/MD.0000000000004165

Conflict of Interest: The authors declare that the research was conducted in the absence of any commercial or financial relationships that could be construed as a potential conflict of interest.

Publisher's Note: All claims expressed in this article are solely those of the authors and do not necessarily represent those of their affiliated organizations, or those of the publisher, the editors and the reviewers. Any product that may be evaluated in this article, or claim that may be made by its manufacturer, is not guaranteed or endorsed by the publisher.

Copyright $\odot 2022$ Mei, Feng, Feng, Yan, Zhu, Li and Zhu. This is an open-access article distributed under the terms of the Creative Commons Attribution License (CC BY). The use, distribution or reproduction in other forums is permitted, provided the original author(s) and the copyright owner(s) are credited and that the original publication in this journal is cited, in accordance with accepted academic practice. No use, distribution or reproduction is permitted which does not comply with these terms. 